\title{
Anisotropic diffusion in FePt thin films
}

\author{
F. Gröstlinger, ${ }^{1,}{ }^{*}$ M. Rennhofer, ${ }^{1, \dagger}$ M. Leitner, ${ }^{1, \dagger}$ E. Partyka-Jankowska, ${ }^{1}$ B. Sepiol, ${ }^{1}$ B. Laenens, ${ }^{2}$ \\ N. Planckaert, ${ }^{2}$ and A. Vantomme ${ }^{2}$ \\ ${ }^{1}$ Faculty of Physics, University of Vienna, Strudlhofgasse 4, 1090 Vienna, Austria \\ ${ }^{2}$ K. U. Leuven Instituut voor Kern-en Stralingsfysica, Celestijnenlaan 200d, 3001 Heverlee, Belgium
}

(Received 12 December 2011; published 11 April 2012)

\begin{abstract}
A complete picture of $\mathrm{Fe}$ self-diffusion in epitaxial $\mathrm{L} 1_{0}$-ordered $\mathrm{FePt}$ thin films is presented. Isotopic multilayers of $\left[{ }^{57} \mathrm{FePt} /{ }^{\text {nat }} \mathrm{FePt}\right]_{10}$ on $\mathrm{MgO}(110)$ were annealed at temperatures between 653 and $743 \mathrm{~K}$. Nuclear resonant scattering reveals the decay of the isotopic superstructure due to diffusion. The well-defined orientation of the lattice in FePt-thin films allows us to resolve the strong anisotropy of diffusion in the tetragonal structure. The diffusion along the $a$-axes of the $\mathrm{L}_{0}$ structure is up to two orders of magnitude faster than along the $c$-axis, which is in line with the commonly assumed diffusion mechanism in this structure. The anisotropy is therefore much higher than in comparable structures such as TiAl. An important subtopic of the study is to compare the classical kinematical evaluation to an analysis employing the full dynamical scattering theory including hyperfine parameters of the nuclear resonances.
\end{abstract}

DOI: 10.1103/PhysRevB.85.134302

PACS number(s): 66.30.Fq, 75.50.Bb

\section{INTRODUCTION}

In our information society stable high-density data storage is of great importance. Various materials are being investigated as storage media for such devices. Two promising approaches for next-generation storage devices have been identified: perpendicular data recording in thin films ${ }^{1,2}$ and patterned media of monodisperse nanoparticles. ${ }^{3-5}$ In both cases FePt is one of the most promising candidates, because of the large magnetocrystalline uniaxial anisotropy $\left(\sim 7 \mathrm{~J} \mathrm{~cm}^{-3}\right)$ of the $\mathrm{L}_{0}$ phase in general ${ }^{6}$ and the thermal stability of this material in particular. ${ }^{7}$ The tetragonal $\mathrm{L} 1_{0}$ phase of FePt exists over a wide compositional range, from 0.45 to 0.65 atomic fraction of $\mathrm{Fe}$, and remains ordered up to $1553 \mathrm{~K}$. Information about atomic motion in thin films is crucial for their synthesis and stability. This calls for a detailed investigation of the fundamental mechanism of diffusion at temperatures as low as possible.

The standard method for determining macroscopic diffusion coefficients in solids is the tracer technique. ${ }^{8}$ By dramatically decreasing the length scales in the samples and employing x-ray reflectometry, this basic idea is applicable at slower diffusivities (e.g., at lower temperatures). Although it would be possible to extract the full information from scattering on a single interface, multilayers are used to amplify the essential parts of the scattering experiment by superposition, thus increasing the contrast of the experiment. So analogously to Bragg scattering, the layered superstructure generates intensity maxima at rather low angles corresponding to the large superstructure period in the film. Additionally, for an approximately periodic structure a simple expression for the evaluation in terms of diffusion can be found in Eq. (5). This concept was first proven valid for interdiffusion in chemically inhomogeneous multilayers. ${ }^{9}$ To study self-diffusion in chemically homogeneous solids, nuclear sensitive methods have to

Published by the American Physical Society under the terms of the Creative Commons Attribution 3.0 License. Further distribution of this work must maintain attribution to the author(s) and the published article's title, journal citation, and DOI. be introduced. Besides neutron reflectometry, ${ }^{10}$ it is possible to utilize the well-known nuclear transition of $14.4 \mathrm{keV}$ of the Mössbauer isotope ${ }^{57} \mathrm{Fe}$ for nuclear resonant scattering. ${ }^{11}$ For this purpose samples with a one-dimensional superstructure are required, preferably of a period just about one order of magnitude above the lattice constant. These features are best achieved by films with an isotopic substructure in depth. ${ }^{12,13}$ In addition, the orientation of the lattice can be chosen by a suitable substrate, thus enabling the measurement of diffusion in different directions and therefore resolving its anisotropy.

Assuming the kinematical approximation for the scattering process, one can derive a direct relation between the decay of the superstructure Bragg peak intensity of a multilayer and the diffusion constant. ${ }^{9}$ In this article, however, we apply the full dynamical scattering theory including nonperiodic boundary conditions, the distinct hyperfine parameters, and the integration time window of the detector.

\section{THEORY}

The appropriate theoretical framework for describing the temporal evolution of a sample with a layered concentration profile $c$ is simply the one-dimensional diffusion equation with constant diffusion coefficients $D$ :

$$
\left(\partial_{t}-D \partial_{x}^{2}\right) c(t, x)=0 .
$$

Note that, in contrast to diffusion in chemically inhomogeneous systems, the chemical composition is uniform here, therefore no concentration dependence of $D$ complicates the picture.

For the classical interpretation of scattering from diffusing concentration profiles, the diffusion equation, (1), is solved via Fourier expansion of the concentration $c$ :

$$
c(t, x)=\sum_{m} C_{m}(t) e^{i q_{m} x} .
$$

In this way one finds an exponential decay of the spatial Fourier coefficients of the concentration profile $C_{m}$ as a function of 
time $t$,

$$
C_{m}(t)=C_{m}(0) e^{-q_{m}^{2} D t},
$$

with the scattering vector of the Bragg peak $q_{m}$. The scattered intensity $I$ in the kinematical approximation is again related to the Fourier transform of the concentration,

$$
I(t, q)=|A(t, q)|^{2} \propto\left|\int d x c(t, x) e^{-i q x}\right|^{2},
$$

with the scattering amplitude $A$. Based on these assumptions, DuMond and Yout ${ }^{9}$ derived a simple relation between the decay of the intensity $I$ of the superstructure peak at a scattering vector $q_{m}$ and the elapsed diffusion time $t$,

$$
\ln \frac{I\left(t, q_{m}\right)}{I\left(0, q_{m}\right)}=\ln \left|\frac{C_{m}(t)}{C_{m}(0)}\right|^{2}=-2 q_{m}^{2} D t,
$$

allowing for direct access to the diffusion constant $D$.

Analogous to Bragg scattering, the layered superstructure generates intensity maxima at rather low angles corresponding to the large superstructure period in the film. The Laue condition $\boldsymbol{q} \cdot \boldsymbol{x}=2 \pi \mathrm{m}$ with the scattering vector $\boldsymbol{q}$ yields, for the first-order maximum $m=1$ in the small angle approximation, a scattering angle of $\theta \approx \pi \hbar c / E_{0} x$. For an x-ray energy of $E_{0}=14.4 \mathrm{keV}$ and a superstructure period of $x=4 \mathrm{~nm}$, we obtain $\theta \approx 11.2$ mrad.

Various aspects of the approach as outlined above were recently criticized in connection with this type of experiment. ${ }^{14}$ In particular, the kinematical approximation ceases to hold (i) in the vicinity of the critical angle and (ii) for nuclear resonant scattering. The full dynamical treatment of the scattering including all hyperfine parameters and respective distributions as well as the detection time window is therefore necessary.

The first step is to solve the diffusion equation, (1), with suitable boundary conditions, which in our case is an initial concentration variation $c(0, x)=g(x)$ and Neumanntype boundary conditions at the film surface $x=0$ and the substrate interface $x=d$ [more precisely, $\partial_{x} c(t, 0)=0$ and $\left.\partial_{x} c(t, d)=0\right]$. The fundamental solution $f$ of the form of a normal distribution

$$
f(t, x)=(4 \pi D t)^{-1 / 2} e^{-x^{2} / 4 D t}
$$

may then be used to solve the diffusion equation in the reciprocal domain $q$, because in this way the convolution reduces to a simple multiplication as

$$
c(t, x)=(f * g)(t, x)=\mathcal{F}^{-1}((\mathcal{F} f)(\mathcal{F} g))(t, q),
$$

with the Fourier transform operator $\mathcal{F}$. The Neumann-type boundary conditions for the diffusion equation are included by mirroring the starting composition at one end ensuring full periodicity and vanishing concentration gradient on the boundaries.

The dynamical theory for the calculation of the reflectivity of such layered samples is outlined in Appendix B.

\section{EXPERIMENT}

The small dimensions call for high-quality samples. The bulk lattice constants for the intermetallic tetragonal $\mathrm{L}_{0}$
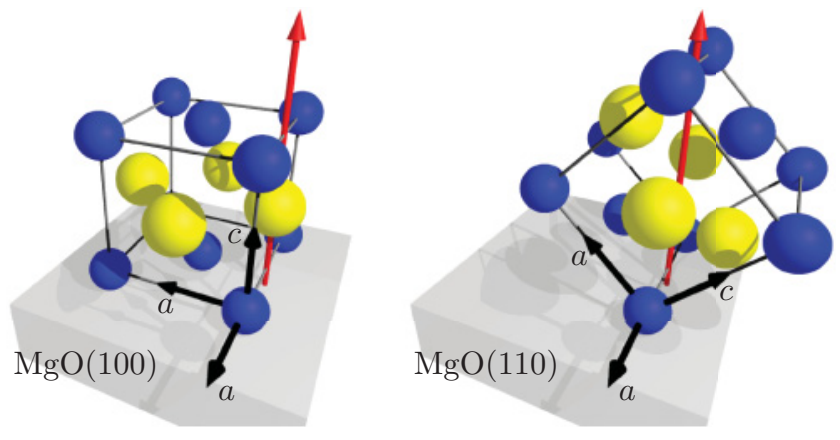

FIG. 1. (Color online) $\mathrm{L}_{0}$-FePt on $\mathrm{MgO}$. (a) $c$ variant on the (100) plane of the previous study; ${ }^{13}$ (b) $45^{\circ}$ canted structure on a (110) plane. The upward-pointing (red) arrow indicates the measured diffusion direction.

phase of FePt are $a_{1}=a_{2}=a=0.38504(8) \mathrm{nm}$ and $a_{3}=$ $c=0.37212(3) \mathrm{nm},{ }^{15}$ respectively, resulting in a lattice misfit of $9 \%$ on a $\mathrm{MgO}(001)$ surface $(0.421 \mathrm{~nm})$. The induced strain is often reduced by various buffer layers (i.e., $\mathrm{Pt}$ ). To avoid island growth ${ }^{16}$ and guarantee a flat film surface, however, the FePt films for this study are directly grown on $\mathrm{MgO}$ under well-tested conditions. ${ }^{17}$ Unfortunately it is not possible to prepare a film with the magnetic main axis, which is along the $c$ lattice direction, purely parallel to the film surface ( $a$ variant) on $\mathrm{MgO}(001)$ at low enough temperatures to avoid dilution of the isotopic multilayers, as it is for the $c$ variant (perpendicular). ${ }^{13}$ On $\mathrm{MgO}(110)$, however, Laenens et al. found a way to prepare FePt films with the structure rotated about one of the $a$-axes by $45^{\circ}$. From this orientation, shown in Fig. 1(b), and the results on diffusion in the $c$-direction in Ref. 13, we can deduce the diffusion constant in the $a$-direction and complete the picture of Fe self-diffusion in FePt.

The samples were prepared by molecular beam epitaxy at a substrate temperature of $623 \mathrm{~K}$ and a pressure $p<$ $10^{-10} \mathrm{mbar}$. The layer composition is $\left[{ }^{57} \mathrm{FePt}(1.6 \mathrm{~nm}) /{ }^{\text {nat }} \mathrm{FePt}\right.$ $(2.4 \mathrm{~nm})]_{10} / \mathrm{MgO}(110)$. The true chemical composition of $\mathrm{Fe}_{56} \mathrm{Pt}_{44}$ determined by Rutherford backscattering, although on the Fe-rich side, is still well in the center of the $\mathrm{L} 1_{0}$ phase.

An x-ray diffraction scan on the as-prepared samples with the scattering vector perpendicular to the surface reveals intensity maxima at Bragg angles of $16.48^{\circ}$ and $34.77^{\circ}$ for the (110) and (220) plane of FePt, respectively, besides the $\mathrm{MgO}(220)$ peak at $31.15^{\circ}$. On the one hand, from the $\operatorname{FePt}(220)$ diffraction we can conclude that the (220) planes are in fact grown parallel to the surface. On the other hand, however, the sheer existence of the (110) peak also implies that in part of the film the $c$-axis is oriented parallel to the surface, which translates to domains, where the measured superstructure decay corresponds to diffusion purely parallel to planes spanned by the $a$-axes.

These results are also confirmed by conversion electron Mössbauer spectroscopy (CEMS) measurements analogous to those in Ref. 18. In an $\mathrm{L}_{0}$ lattice the main axis of the magnetic hyperfine field is always parallel to the $c$-axis. ${ }^{19}$ The relative intensity of the second and fifth to the inner resonances 3: $f(\theta): 1: 1: f(\theta): 3$ is directly related to the angle $\theta$ between the wave vector of the incoming photon and the direction of the magnetic hyperfine field, which is the $c$-direction in the 


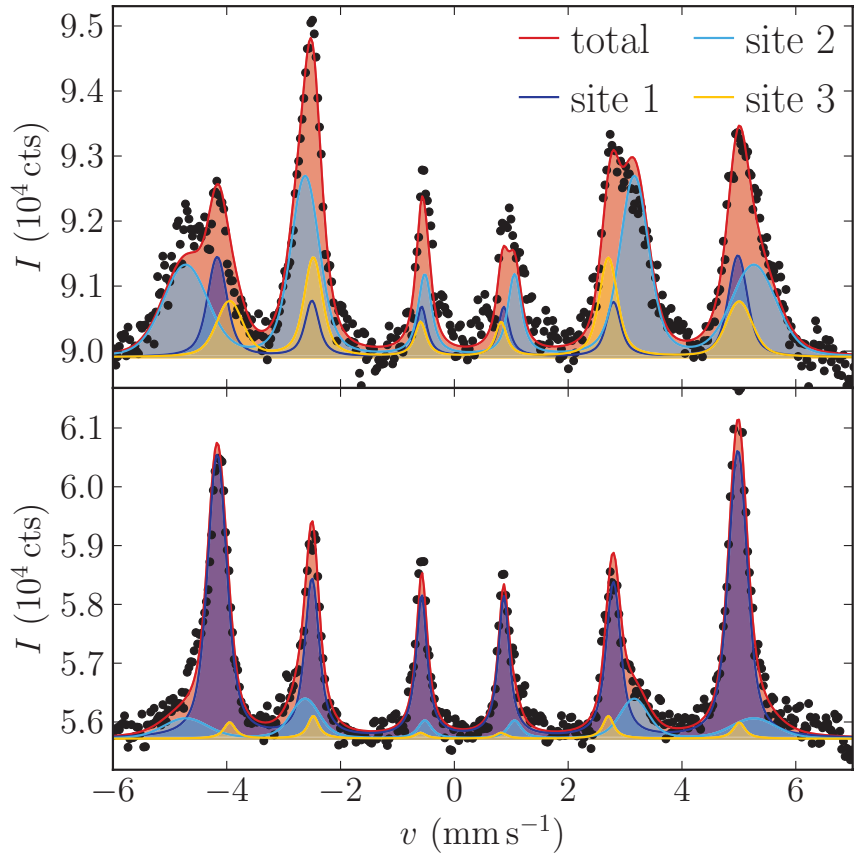

FIG. 2. (Color online) CEMS spectra of the FePt samples. The as-prepared sample (top) shows significant fractions of domains with different orientation. The measured samples (bottom) show good ordering, with the majority of domains (site 1 ) having the $c$-axis oriented $45^{\circ}$ to the incoming photons ( $\perp$ surface). In the domains of the other two sites with different hyperfine parameters the $c$-axis is oriented parallel to the surface.

tetragonal structure, by

$$
f(\theta)=\frac{4 \sin ^{2} \theta}{1+\cos ^{2} \theta} .
$$

The acquired spectra are fitted using three independent sites by a Voigt-based method for arbitrary distribution of magnetic hyperfine fields ${ }^{20}$ displayed in Fig. 2. The hyperfine parameters are simultaneously fitted for all samples, as they are parts of one large, homogeneously prepared sample. Only the standard deviation of the normal distribution of the magnetic hyperfine field of site 3 of the as-prepared sample T1 was decoupled to account for higher disorder and resulted in $\sigma_{B}=1.11 \mathrm{~T}$. The other parameters are listed in Table I. Besides the as-prepared one, the samples show the majority, on average, a fraction of $w=0.72$, of domains with a $45^{\circ}$ canted structure as intended for the experiment. The other two sites suggest so called $a$ variant domains with the magnetic axis parallel to the surface. In this fraction the diffusion constant is thus purely measured in the $a$-direction. The majority, however, contributes with a reduced diffusion constant, discussed in more detail below. The resulting hyperfine parameters are finally used in the calculation of the nuclear resonant reflectivity.

The scattering experiment was conducted at beamline ID22N of the ESRF, which is optimized for nuclear resonant scattering. ${ }^{21}$ The samples were successively annealed at a fixed temperature $(653,673,698,723$, or 743$) \mathrm{K}$ in a quartz-tube furnace in a vacuum of better than $10^{-6}$ mbar. After the heat treatment the samples were pulled out of the furnace to essentially freeze the diffusion. The nuclear resonant $\Theta-2 \Theta$ scan was accumulated ex situ on the beamline under ambient conditions. The simultaneously collected electronic reflectivity revealed an overall thickness of the isotopic multilayer of $39.7 \mathrm{~nm}$.

The delayed nuclear reflectivity for each scattering vector $q$ is acquired over a time window of $170 \mathrm{~ns}$ after the $\mathrm{x}$-ray illumination, which is roughly the time between two electron bunches in the 16-bunch mode of the synchrotron. The intensity is integrated starting at $15 \mathrm{~ns}$ to avoid any disturbance by the prompt signal due to the electronic reflectivity, which is orders of magnitude more intense. ${ }^{21}$ The measured nuclear reflectivities for successive annealing steps are illustrated in Fig. 3 (top) for the sample annealed at $698 \mathrm{~K}$.

\section{RESULTS}

The solution of dynamical scattering of Eq. (B3) is only valid for stacked homogeneous layers. Hence, one has to discretize the continuous solution of the diffusion equation into a sufficiently large number of homogeneous layers (in our case, 100) with varying enrichment of the ${ }^{57} \mathrm{Fe}$ isotope. As mentioned above, the Neumann-type boundary conditions for the diffusion equation are included by mirroring the starting composition at one end. In general, the problem of dynamical scattering on a layered film [Eq. (B1)] is only numerically solvable; to this end, we use the program package CONUSS. $^{22,23}$ All parameters of the nuclear resonances, the hyperfine magnetic field, and its weights, orientations, and distributions derived from CEMS are considered accordingly. The choice of the start of the detection window in the range of 0-20 ns did not influence the results for our samples, as it basically only scaled the overall intensity.

Besides the intensity normalization, the product $D t$, called the diffusion progress throughout this article, is the only free parameter in fitting the calculated reflectivity to the experimental data. The mean square deviation of the calculated reflectivities from the measured ones is minimized via a Nelder-Mead algorithm to find the best estimator for $D t$. This

TABLE I. CEMS parameters for the three sites. All the hyperfine parameters, i.e., the isomer shift (IS), the quadrupol splitting (QS), and the magnetic hyperfine field $B$ and its distribution $\sigma_{B}$, were simultaneously fitted for all the samples, except the magnetic field distribution of the first sample was decoupled to account for higher disorder. The fractions of the different sites are listed.

\begin{tabular}{|c|c|c|c|c|c|c|c|c|c|c|c|}
\hline \multirow[b]{2}{*}{ Site } & \multirow{2}{*}{$\begin{array}{c}\text { IS } \\
\left(\mathrm{mm} \mathrm{s}^{-1}\right)\end{array}$} & \multirow{2}{*}{$\begin{array}{c}\mathrm{QS} \\
\left(\mathrm{mm} \mathrm{s}^{-1}\right)\end{array}$} & \multirow{2}{*}{$\begin{array}{c}B \\
(\mathrm{~T})\end{array}$} & \multirow{2}{*}{$\begin{array}{l}\sigma_{B} \\
(\mathrm{~T})\end{array}$} & \multirow{2}{*}{$\begin{array}{c}\theta \\
(\operatorname{deg})\end{array}$} & \multicolumn{6}{|c|}{ Fraction of sites by sample } \\
\hline & & & & & & $\mathrm{T} 1$ & $\mathrm{~T} 2$ & $\mathrm{~T} 3$ & $\mathrm{~T} 4$ & T6 & $\mathrm{T} 8$ \\
\hline 1 & 0.277 & 0.207 & 28.36 & 0.68 & 45 & 0.28 & 0.65 & 0.84 & 0.69 & 0.77 & 0.68 \\
\hline 2 & 0.269 & 0.000 & 30.97 & 2.13 & 90 & 0.52 & 0.25 & 0.12 & 0.23 & 0.18 & 0.26 \\
\hline 3 & 0.321 & 0.207 & 27.76 & 0.20 & 90 & 0.20 & 0.10 & 0.04 & 0.08 & 0.05 & 0.06 \\
\hline
\end{tabular}



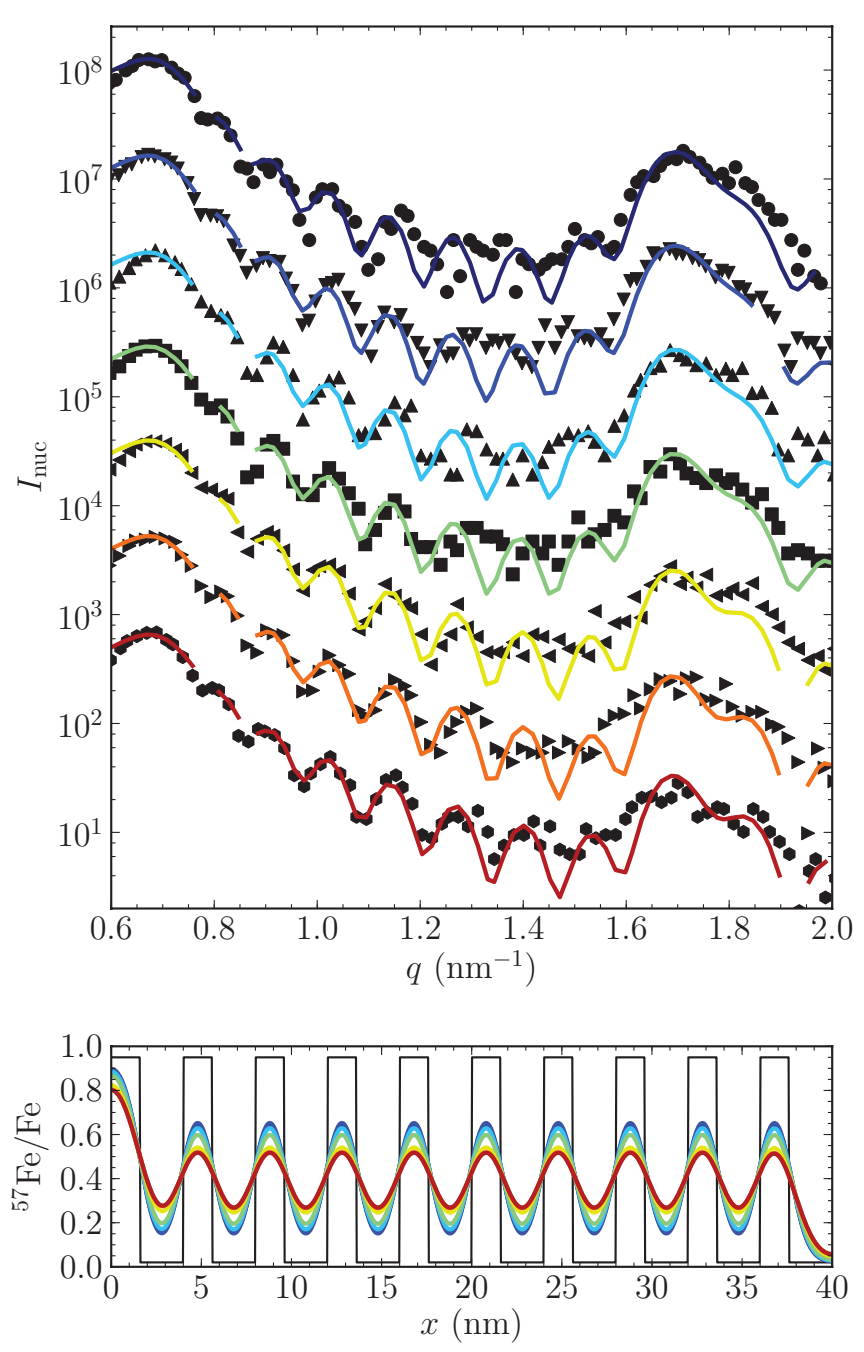

FIG. 3. (Color online) Nuclear resonant reflectivity $I_{\text {nuc }}$ for the ${ }^{57} \mathrm{FePt} / \mathrm{FePt}$ multilayer at $698 \mathrm{~K}$ versus scattering vector $q$ for increasing annealing time from top to bottom. Data (filled symbols) were shifted for better visibility. Colored lines are the calculated dynamical nuclear reflectivities corresponding to the discretized solutions of the diffusion equation for the enrichment of ${ }^{57} \mathrm{Fe}$ at depth $x$ of the film as shown below.

procedure is repeated for every sample. The fits established in this way are drawn in Fig. 3 for the sample annealed at $98 \mathrm{~K}$. The diminishing nuclear Bragg peak clearly corresponds to the decrease in the variation of the tracer enrichment in the sample. The high-frequency oscillation reflects the total thickness of the multilayer and is not affected by isotopic diffusion. From the slight deviation of the experimental data one can conclude that there is, however, some sort of composition imperfection of the samples which could not be resolved by our model.

For a certain temperature the estimator for $D t$ is plotted versus the time duration of the annealing $t$ (Fig. 4). In this representation the slope of a straight line fitted to the data is the diffusion constant $D$. Because of the elevated temperature and enhanced mobility in the vicinity of the surface during sample preparation, the initial composition is not perfectly step-like. The initial state of the samples should be the same, as they are cut from one large homogeneous sample, therefore we require the intersection of all the fitted lines at the ordinate.

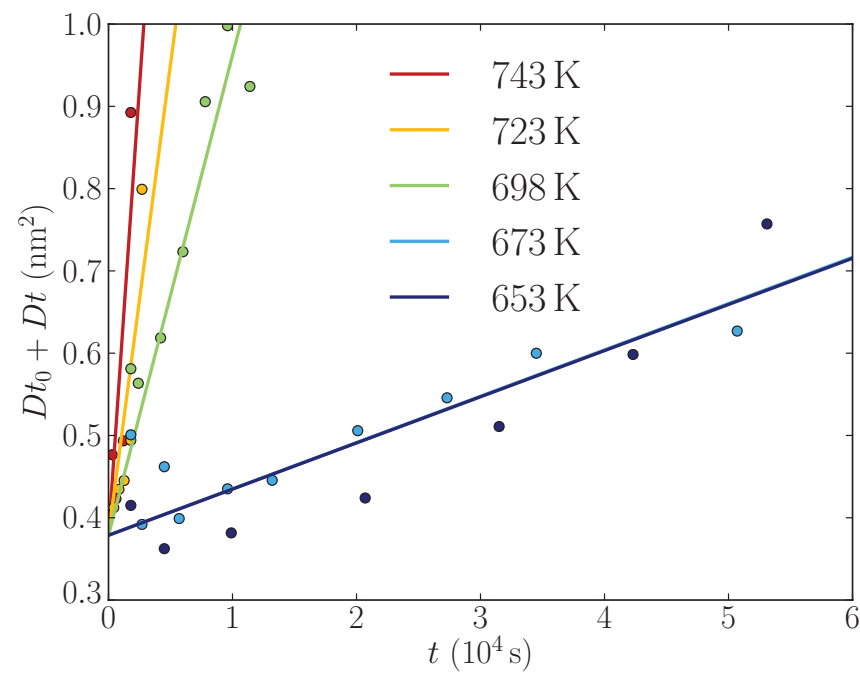

FIG. 4. (Color online) The fitted diffusion progress $D t$ plotted versus the anneal time $t$ for all temperatures, together with a linear fit. The slope of the fitted lines provides the diffusion constant.

In our case this joint initial diffusion progress is $D t_{0}=0.3787$ $\mathrm{nm}^{2}$. The resulting values for $D$ are listed in Table II.

In the case of the dynamical scattering evaluation the two domains with fractions $w$ and $1-w$ have been incorporated in the theory by two different normal distributions of Eq. (6) with $D_{1}=\left(D_{a}+D_{c}\right) / 2 \approx D_{a} / 2$, assuming $D_{c} \ll D_{a}$, and $D_{2}=$ $D_{a}$ for $D$, respectively. On the other hand, the kinematical evaluation according to Eq. (5) can be corrected in a similar manner. If the domains of the different sites are assumed to be distributed equally over the depth of the sample, we may solve the diffusion equation for two independent atom groups. Keeping in mind that in domains of site 2 and site 3 the $c$-axis is parallel to the surface, the diffusion direction is the same and we can treat them as one domain. In this picture the scattered intensity in the kinematic approximation is

$$
I(t, q) \propto\left|\int d x e^{-i q x}\left(w c_{1}(t, x)+(1-w) c_{2}(t, x)\right)\right|^{2} .
$$

Expanding the individual concentrations using Eqs. (2) and (3) and assuming an equal initial composition $C_{m 1}(0)=$ $C_{m 2}(0) \equiv C_{m}(0)$, we arrive at an adapted expression (see Appendix A) for the logarithmic relative intensity decay over time in comparison to Eq. (5) for $D_{1}=D_{a} / 2, D_{2}=D_{a}$, and

TABLE II. Total annealing time $t$ and diffusion constants for kinematical and dynamical evaluation, $D_{\text {kin }}$ and $D_{\text {dyn }}$, respectively, for different annealing temperatures $T$.

\begin{tabular}{lcccc}
\hline \hline & $\begin{array}{c}T \\
\text { Sample }\end{array}$ & $\begin{array}{c}t \\
\left(10^{3} \mathrm{~s}\right)\end{array}$ & $\begin{array}{c}D_{\text {kin }} \\
\left(10^{-22} \mathrm{~m}^{2} \mathrm{~s}^{-1}\right)\end{array}$ & $\begin{array}{c}D_{\text {dyn }} \\
\left(10^{-22} \mathrm{~m}^{2} \mathrm{~s}^{-1}\right)\end{array}$ \\
\hline T4 & 653 & 53.1 & $0.064(10)$ & $0.056(11)$ \\
T2 & 673 & 50.7 & $0.060(9)$ & $0.056(12)$ \\
T3 & 698 & 11.4 & $0.505(38)$ & $0.583(47)$ \\
T6 & 723 & 2.7 & $1.65(35)$ & $1.14(23)$ \\
T8 & 743 & 1.8 & $2.79(80)$ & $2.18(36)$ \\
\hline \hline
\end{tabular}




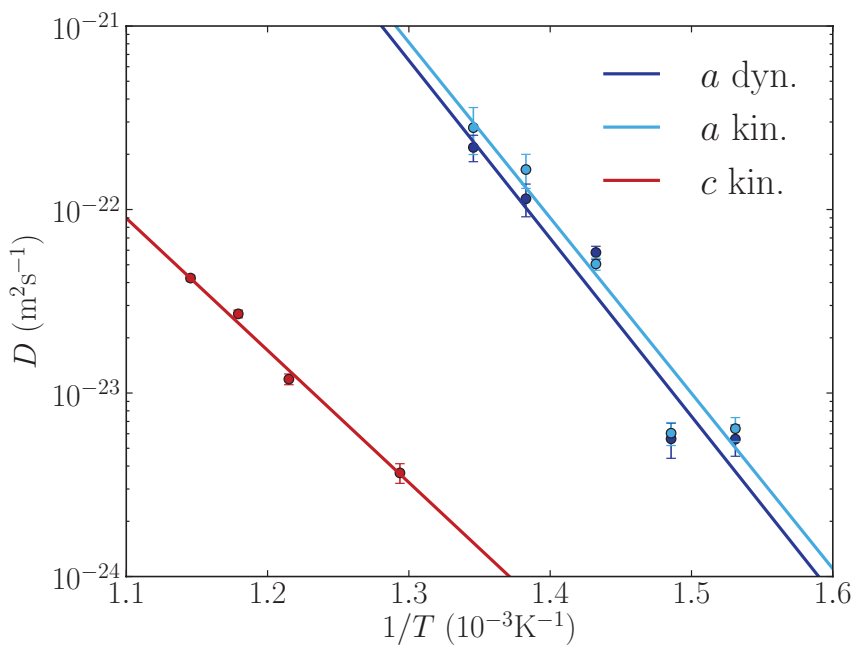

FIG. 5. (Color online) Arrhenius plot of diffusion constant $D$ versus inverse temperature $T$ for the $c$-direction ${ }^{13}$ and $a$-direction, evaluated by kinematical and dynamical scattering theory, respectively. The slope represents the activation energy of the diffusion jumps resulting in 1.90(23) and 1.92(23) eV for kinematical and dynamical evaluation, respectively, compared to the previously measured $1.65(29) \mathrm{eV}^{13}$

$$
\begin{aligned}
& 0 \leqslant w \leqslant 1, \\
& \begin{aligned}
\ln \frac{I(t, q)}{I(0, q)} & =2 \ln \sum_{i} w_{i} e^{-q^{2} D_{i} t} \\
& =2 \ln \left(w e^{-q^{2} D_{1} t}+(1-w) e^{-q^{2} D_{2} t}\right) \\
& =-q^{2} D_{a} t+2 \ln \left(w+(1-w) e^{-q^{2} D_{a} t / 2}\right),
\end{aligned}
\end{aligned}
$$

which considers the smaller effective diffusion constant measured for the majority domain canted to the actual diffusion in the $a$-direction.

Finally, the activation energies of the fundamental diffusion jump can be extracted from the Arrhenius plot in Fig. 5. Here the diffusion constant is plotted versus the inverse temperature and compared with the conventional kinematical evaluation along the same direction and the diffusivity in the perpendicular direction.

\section{DISCUSSION}

The difference in the activation energies 1.90(23) eV (kinematical evaluation) and 1.92(23) eV (dynamical evaluation) is negligible and well in the range of the standard deviation. Therefore we can basically confirm the results of the kinematic evaluation. Additionally, the diffusion constants are only about $20 \%$ lower for the dynamical evaluation in the range examined here. Interestingly, the activation energies are in the same region as the previously measured one $[1.65(29) \mathrm{eV}]$ in the perpendicular direction.

These values for the activation energy are lower compared to Fe-tracer ${ }^{24,25}$ or chemical $^{26}$ diffusion data in FePt, which were, however, obtained at much higher temperatures. Lower activation energies are usually explained by grain boundary diffusion with a high concentration of point defects. ${ }^{27}$ Due to the probed length scales, which are barely an order of magnitude above the lattice constant, only an extremely high density of such defects would be able to influence the diffusivities measured with our method, which is in noted contrast to the case of conventional tracer diffusion experiments. The high epitaxial quality of our samples, however, is confirmed by TEM images ${ }^{17}$ for the $c$-variant samples, revealing antiphase boundaries and dislocations mainly at the interface to the substrate. Furthermore, the dislocation lines are oriented perpendicular to the measured diffusion. Due to the good agreement in the activation energies of the $c$ variant, $1.65(29) \mathrm{eV},{ }^{13}$ and the direction investigated here, we may disregard these influences in this study. The rather low activation energy is also confirmed by in situ resistometry measurements on a similar FePt isotopic multilayer by Kozubski et al. ${ }^{28}$ Additionally, they observed a similar multiscale character of the activation energies in order-order transitions investigated by Monte Carlo simulations.

To recapitulate, we believe that the activation energy in our measurements on two different samples so far and in different crystallographic directions has a solid base experimentally. New tracer or interdiffusion measurements filling the huge gap in the temperature between our data and the data in Ref. 24 would therefore be desirable.

The Fe diffusion rate parallel to the $a$ plane, which is enhanced by up to two orders of magnitude compared to the diffusion rate along the $c$-axis, qualitatively confirms the expectation of diffusion mainly in the Fe sublattice. However, the anisotropy is surprisingly high compared with that of other intermetallics of the same structure.

Anisotropic diffusion measurements are very rare for $\mathrm{L} 1_{0}$ single crystals. Studies on $\mathrm{L}_{0}-\mathrm{FePt}(0.54$ atomic fraction of $\mathrm{Fe}$, close to the fraction of 0.56 of our sample) report an anisotropy of only 1.2 to 1.7 , i.e., faster iron diffusion along the $a$ than along the $c$-axis. ${ }^{24,25}$ This value increases only slightly, to a factor of 1.6 to 3.6 , in the Pt-rich composition with 0.42 atomic fraction of Fe. The anisotropy of Ti diffusion in $\mathrm{L} 1_{0}-\mathrm{TiAl}$ is 1 order of magnitude. ${ }^{29,30}$ Anisotropies of impurity diffusion in $\mathrm{TiAl}^{31}$ can be larger as well as smaller than 1 , which proves that activation energies play a crucial role in the case of impurities.

In the case of perfect stoichiometry we assume that any Fe diffusion in the $c$-direction will require higher energy due to the creation of $\mathrm{Pt}$ antisites, and therefore the energetically favored diffusion paths are exclusively in the $a$ planes. The raw formation enthalpies from $a b$ initio calculations ${ }^{32}$ for $\mathrm{Fe}$ $(\mathrm{Pt})$ antisites and vacancies, 1.05 (0.66) and 2.58 (3.2) eV, respectively, can be renormalized for structural antisites ${ }^{33}$ in the Fe-rich composition to the effective formation enthalpies of 0 (1.71) and $3.105(2.675) \mathrm{eV}$, respectively, to achieve the right kinetics. For diffusion in the $c$-direction, diffusion paths via the classical six-jump cycle ${ }^{34}$ (see also Mehrer $^{8}$ ) and direct jumps to a next-nearest neighbor site are both energetically disadvantageous. The very high order of the FePt phase clearly supports this view. In our slightly off-stoichiometric Fe-rich samples a small fraction of diffusion paths of similar energetics to the $a$ planes through structural Fe antisites exists. Nevertheless, in our opinion the reason for the high anisotropy is the far higher number of energetically favoured diffusion paths on the atoms own sublattice. These are diffusion jumps which in no way disturb the lattice order.

Additional diffusion paths through $\mathrm{Fe}$ antisites would, however, mainly influence diffusion in the $c$-direction, which was measured on samples closer to stoichiometry. Despite 
their far lower numbers, the good agreement of the activation energy in the different directions suggests that diffusion in the $c$-direction similarly occurs through antisites on the Pt sublattice, which could be another reason for the high anisotropy. Nevertheless, from these considerations we expect even higher anisotropy for Pt. Overall, the presence of antisites could decrease the formation energy of vacancies considerably and calls for further $a b$ initio calculations.

There is one more factor acting in the same way as energetically favored diffusion paths: the correlation effect for the vacancy jump as a diffusion vehicle. In the paper by Ikeda et al. ${ }^{29}$ the smaller diffusion coefficient of $\mathrm{Ti}$ in the direction parallel to the $c$-axis was explained in terms of the stronger correlation effect for vacancy jumps between sublattices. This claim was modified in the later paper, ${ }^{25}$ but certainly some part of the anisotropy could also be understood by taking only vacancy correlation effects into account.

In summary, anisotropy of iron diffusion in $\mathrm{L}_{0}-\mathrm{FePt}$ is a result of energetic barriers and vacancy correlations. We can, however, not separate the relative impacts of all these factors.

Taking into account all these factors, we conclude that $\mathrm{L} 1_{0}$-FePt displays the highest anisotropy of the iron diffusion coefficient in the $c$ vs perpendicular to the $c$-axis among all $\mathrm{L}_{0}$ systems reported so far. We cannot conclude which factor is the most important and leave this problem as a challenge for future ab initio calculations.

A full dynamical treatment for multilayer diffusion incorporating all the hyperfine parameters was employed to a real resonant reflectivity experiment. Within the variance the results are in good agreement with the classical evaluation method. In samples with a higher ratio of nuclear resonant absorption to photoabsorption, however, significant deviations can be seen, which will be the object of a different publication.

\section{APPENDIX A: KINEMATICAL EVALUATION FOR MULTIPLE DOMAINS}

In single crystals of a tetragonal lattice there are always three possible orientations for the different domains. For anisotropic diffusion, Eq. (5) has to be modified. Approximately equally distributed domains of concentration $c_{i}$ and sizes smaller than the transversal coherence length can be weighted by the abundance $w_{i}$ and summed over coherently, proceeding as

$$
\begin{aligned}
I(t, q) & \propto\left|\int d x e^{-i q x} \sum_{i} w_{i} c_{i}(t, x)\right|^{2} \\
& =\left|\int d x e^{-i q x} \sum_{i} w_{i} \sum_{m} C_{m i}(0) e^{-q_{m}^{2} D_{i} t} e^{i q_{m} x}\right|^{2} \\
& =\left|\sum_{i} w_{i} \sum_{m} C_{m}(0) e^{-q_{m}^{2} D_{i} t} \int d x e^{-i\left(q-q_{m}\right) x}\right|^{2} \\
& =\left|\sum_{i} w_{i} \sum_{m} C_{m}(0) e^{-q_{m}^{2} D_{i} t} \delta\left(q-q_{m}\right)\right|^{2} \\
& =\left|C_{q}(0)\right|^{2}\left|\sum_{i} w_{i} e^{-q^{2} D_{i} t}\right|^{2}
\end{aligned}
$$

where we assumed an approximately equal starting composition throughout the domains $C_{m i}(0) \equiv C_{m}(0)$. For a certain wave vector $q$, typically the first Bragg peak of the superstructure, and the abundance normalization $\sum_{i} w_{i}=1$, we may further write

$$
\begin{aligned}
\ln \frac{I(t, q)}{I(0, q)} & =\ln \frac{\left|C_{q}(0)\right|^{2}\left|\sum_{i} w_{i} e^{-q^{2} D_{i} t}\right|^{2}}{\left|C_{q}(0)\right|^{2}\left|\sum_{i} w_{i}\right|^{2}} \\
& =2 \ln \sum_{i} w_{i} e^{-q^{2} D_{i} t} .
\end{aligned}
$$

Unfortunately in this case there is no longer a strict linear dependence on $t$, so nonlinear fitting algorithms, i.e., LevenbergMarquardt, have to be applied to extract the diffusion constants $D_{i}$.

\section{APPENDIX B: NUCLEAR RESONANT SCATTERING}

The problem of dynamical scattering on an in-plane infinite, homogeneous but in-depth $x$ varying layer can always be written in the form

$$
\partial_{x} \boldsymbol{A}(x)=i \boldsymbol{F}(x) \boldsymbol{A}(x),
$$

with the electric field amplitudes $\boldsymbol{A}$ and the propagation matrix $\boldsymbol{F}$. For a homogeneous layer of thickness $d$ the solution for this system of coupled linear differential equations is

$$
\boldsymbol{A}(d)=e^{i \boldsymbol{F} d} \boldsymbol{A}(0) .
$$

Therefore the solution for $N$ homogeneous layers with the individual propagation matrices $\boldsymbol{F}_{j}$ and thicknesses $d_{j}$ and the overall thickness $d=d_{1}+\cdots+d_{N}$ is

$$
\boldsymbol{A}(d)=e^{i \boldsymbol{F}_{N} d_{N}} \cdots e^{i \boldsymbol{F}_{1} d_{1}} \boldsymbol{A}(0) \equiv \boldsymbol{S} \boldsymbol{A}(0) .
$$

In this way the scattering amplitude of an in-depth, slowly varying layer can be calculated in a good approximation by dividing it into a large number of sublayers, applying dynamical scattering theory to the individual layer. A similar approach with infinitesimal layers led to Eq. (B1) in the first place. The discretization is done by an algorithm repositioning the layer interfaces to minimize the squared deviation to the continuous solution of the diffusion equation.

If the propagation equation, (B3), with the total scattering matrix $S$ is written in matrix form,

$$
\left[\begin{array}{l}
\boldsymbol{A}_{+}(d) \\
\boldsymbol{A}_{-}(d)
\end{array}\right]=\left[\begin{array}{ll}
\boldsymbol{S}_{++} & \boldsymbol{S}_{+-} \\
\boldsymbol{S}_{-+} & \boldsymbol{S}_{--}
\end{array}\right]\left[\begin{array}{l}
\boldsymbol{A}_{+}(0) \\
\boldsymbol{A}_{-}(0)
\end{array}\right],
$$

then $\boldsymbol{A}_{+}$and $\boldsymbol{A}_{-}$denote the field amplitudes propagating into and out of the sample, respectively. The components are themselves two-dimensional vectors and matrices to account for polarization dependence. After applying the boundary condition that there is no incident wave to the bottom of the sample $\boldsymbol{A}_{-}(d)=0$, the reflected amplitude is given by

$$
\boldsymbol{A}_{-}(0)=-\boldsymbol{S}_{--}{ }^{-1} \boldsymbol{S}_{-+} \boldsymbol{A}_{+}(0) .
$$

This theoretical approach was developed by Batterman and Cole $^{35}$ and extended to nuclear resonant scattering, ${ }^{36}$ which is presented in a more intuitive way for the grazing incidence geometry in Ref. 23. The scattering properties of the individual 
layer $j$ are included in the respective propagation matrix $\boldsymbol{F}_{j}$, consisting of separate electronic and nuclear resonant atomic scattering amplitudes. The nuclear resonant amplitude holds all the hyperfine parameters of the ${ }^{57} \mathrm{Fe}$ atoms in the $\mathrm{FePt}$ structure and is weighted by the enrichment of the individual layer.

\section{ACKNOWLEDGMENTS}

The authors would like to thank S. Stankov of the ESRF for assisting with the experiment. This research was funded by Austrian Science Fund (FWF) Contract Nos. P-20767-N20 and P20713-N20 and by the the K. U. Leuven within the program G0A/09/006.
*Corresponding author: friedrich.groestlinger@univie.ac.at

${ }^{\dagger}$ Present address: Austrian Institute of Technology, Giefinggasse 2, 1210 Vienna, Austria.

${ }^{\ddagger}$ Present address: Forschungsneutronenquelle Heinz Maier-Leibnitz (FRM II), TU München, 85747 Garching, Germany.

${ }^{1}$ M. L. Yan, Y. F. Xu, and D. J. Sellmyer, J. Appl. Phys. 99, 08 G903 (2006).

${ }^{2}$ D. Suess, S. Eder, J. Lee, R. Dittrich, J. Fidler, J. W. Harrell, T. Schrefl, G. Hrkac, M. Schabes, N. Supper, and A. Berger, Phys. Rev. B 75, 174430 (2007).

${ }^{3}$ S. Sun, C. B. Murray, D. Weller, L. Folks, and A. Moser, Science 287, 1989 (2000).

${ }^{4}$ Y. K. Takahashi, K. Hono, T. Shima, and K. Takanashi, J. Magn. Magn. Mater. 267, 248 (2003).

${ }^{5}$ B. D. Terris and T. Thomson, J. Phys. D 38, 199R (2005).

${ }^{6}$ J. B. Staunton, S. Ostanin, S. S. A. Razee, B. Gyorffy, L. Szunyogh, B. Ginatempo, and E. Bruno, J. Phys. Condens. Matter 16, 5623 (2004).

${ }^{7}$ D. Weller, A. Moser, L. Folks, M. E. Best, W. Lee, M. F. Toney, M. Schwickert, J. U. Thiele, and M. F. Doerner, IEEE Trans. Magn. 36, 10 (2000).

${ }^{8}$ H. Mehrer, Diffusion in Solids: Fundamentals, Methods, Materials, Diffusion-Controlled Processes, Springer series in Solid State Science (Springer, Berlin, 2007).

${ }^{9}$ J. DuMond and J. P. Youtz, J. Appl. Phys. 11, 357 (1940).

${ }^{10}$ M. Gupta, A. Gupta, J. Stahn, M. Horisberger, T. Gutberlet, and P. Allenspach, Phys. Rev. B 70, 184206 (2004).

${ }^{11}$ R. Röhlsberger, Nuclear Condensed Matter Physics with Synchrotron Radiation: Basic Principles, Methodology and Applications, Springer Tracts in Modern Physics (Springer, Berlin, 2004).

${ }^{12}$ A. Gupta, M. Gupta, S. Chakravarty, R. Rüffer, H.-C. Wille, and O. Leupold, Phys. Rev. B 72, 014207 (2005).

${ }^{13}$ M. Rennhofer, B. Sepiol, M. Sladecek, D. Kmiec, S. Stankov, G. Vogl, M. Kozlowski, R. Kozubski, A. Vantomme, J. Meersschaut, R. Rüffer, and A. Gupta, Phys. Rev. B 74, 104301 (2006).

${ }^{14}$ M. A. Andreeva, N. G. Monina, and S. Stankov, Moscow Univ. Phys. Bull. 63, 132 (2008).
${ }^{15}$ H. Kudielka and P. Runow, Z. Metallkd. 67, 699 (1976).

${ }^{16}$ M. Kim, S. Shin, and K. Kang, Appl. Phys. Lett. 80, 3802 (2002).

${ }^{17}$ B. Laenens, F. M. Almeida, N. Planckaert, K. Temst, J. Meersschaut, A. Vantomme, C. Rentenberger, M. Rennhofer, and B. Sepiol, J. Appl. Phys. 105, 073913 (2009).

${ }^{18}$ B. Laenens, F. M. Almeida, A. Vantomme, and J. Meersschaut, Acta Phys. Pol. A 112, 1313 (2007).

${ }^{19}$ T. Shinjo and W. Keune, J. Magn. Magn. Mater. 200, 598 (1999).

${ }^{20}$ D. G. Rancourt and J. Y. Ping, Nucl. Instrum. Methods B 58, 85 (1991).

${ }^{21}$ R. Rüffer and A. I. Chumakov, Hyperfine Interact. 97/98, 589 (1996).

${ }^{22}$ W. Sturhahn and E. Gerdau, Phys. Rev. B 49, 9285 (1994).

${ }^{23}$ R. Röhlsberger, Hyperfine Interact. 123/124, 301 (1999).

${ }^{24}$ Y. Nose, T. Ikeda, H. Nakajima, and H. Numakura, Defect Diffus. Forum 237-240, 450 (2005).

${ }^{25}$ H. Nakajima, Y. Nose, N. Terashita, T. Ikeda, and H. Numakura, Defect Diffus. Forum 237-240, 7 (2005).

${ }^{26}$ A. Kushida, K. Tanaka, and H. Numakura, Mater. Trans. 44, 59 (2003).

${ }^{27}$ C.-G. Lee, R. Nakamura, T. Shimozaki, and T. Okino, Defect Diffus. Forum 237-240, 426 (2005).

${ }^{28}$ R. Kozubski, M. Kozlowski, K. Zapala, V. Pierron-Bohnes, W. Pfeiler, M. Rennhofer, B. Sepiol, and G. Vogl, J. Phase Equil. Diffus. 26, 482 (2005).

${ }^{29}$ T. Ikeda, H. Kadowaki, and H. Nakajima, Acta Mater. 49, 3475 (2001).

${ }^{30}$ Y. Mishin, I. V. Belova, and G. E. Murch, Defect Diffus. Forum 237-240, 271 (2005).

${ }^{31}$ Y. Nose, N. Terashita, T. Ikeda, and H. Nakajima, Acta Mater. 54, 2511 (2006).

${ }^{32}$ Y. Sui, Z. Chen, X. Shu, and T. Wang, Mater. Sci. Forum 561-565, 1923 (2007).

${ }^{33}$ B. Meyer and M. Fähnle, Phys. Rev. B 59, 6072 (1999).

${ }^{34}$ E. W. Elcock and C. W. McCombie, Phys. Rev. 109, 605 (1958).

${ }^{35}$ B. W. Batterman and H. Cole, Rev. Mod. Phys. 36, 681 (1964).

${ }^{36}$ M. Blume and O. Kistner, Phys. Rev. 171, 417 (1968). 